\section{SEROLOGICAL DIAGNOSIS OF LYME NEUROBORRELIOSIS IN CHILDREN WITH VARIOUS NEUROLOGICAL DISORDERS - COMPARISON OF TWO ELISA TESTS}

A. Pawinska, E. Vogtt, K. Dzierzanowska-Fangrat Clinical Microbiology, The Children's Memorial Heath Institute, Warsaw, Poland

Aim: We wanted to compare two ELISA kits for detection of IgG and IgM antibodies against Borrelia in samples taken from children with suspected neuroborreliosis.

Methods: Between 01.04.2009 and 31.03.2010 serum and cerebrospinal fluid from children with various neurological symptoms were analyzed using Biomedica kits. Positive samples were confirmed with MIKROGEN Diagnostik Immunoblot assay and tested with DRG Diagnostics kits. The Biomedica IgG kit contains recombinant antigens: OspC, p18, p100 and VIsE ; the IgM Biomedica kit contains recombinant antigens: OspC (B.afzelii, B.garinii), p41/l, VIsE. The DRG IgG kit contains recombinant VIsE antigen and lysate antigens from B.burgdorferi s.s., B.afzelii and B.garinii; the DRG IgM kit contains recombinant $14 \mathrm{kD}$ protein and native OspC. Interpretation of the ELISA and Immunoblot was done according to the manufacturer's instructions.

Results: A total of 379 samples (353 sera and 26 CSF) were analyzed for Borrelia antibodies. Antibodies against B.burgdorferi were detected by Biomedica kit in 55 samples from which 29 (52\%) were confirmed with Immunoblot. Based on the Immunoblot analysis 29 / 379 (7,3\%) samples were considered as "true positive". Among two ELISA tests, 26 sera were false-positive with the Biomedica test and 13 sera were false-positive with the DRG Diagnostics test. However, DRG Diagnostics test failed to detect two sera that were positive with the Biomedica test and Immunoblot.

Conclusions: We observed significant differences between the two ELISA tests. Biomedica test showed higher sensitivity but it produced more false-positive results. DRG Diagnostics test was more specific, but less sensitive.

\section{PLASMA CONCENTRATION OF PYRIMETHAMINE IN INFANTS TREATED FOR CONGENITAL TOXOPLASMOSIS}

B. Lipka¹, B. Milewska-Bobula¹, M. Filipek ${ }^{2}$

${ }^{1}$ Department of Infant Diseases, ${ }^{2}$ Department of Pharmacokinetic, The Children's Memorial Heath Institute, Warsaw, Poland

Aim: Evaluation of the plasma concentration of pyrimethamine (PY) in infants treated for congenital toxoplasmosis.

Material: 20 infants aged from 1 to 5 months, hospitalized from 2006 to 2009.

Methods: Pyrimethamine concentration in plasma was measured before the 6-th dose, then in 4-weeks intervals, and additionally after modification of the dose, using high performance liquid chromatography method. The therapeutic concentration was established as 0,08-0,6 ug/ml. Initial dose of PY was $0.5 \mathrm{mg} / \mathrm{kg}$ given once dialy, simultaneously with sulfadiazine and folinic acid. The peripheral blood cell counts, transaminases, kreatynine and urine were controlled once a week.

Results: 60 tests were performed. The dose of PY varied from 0,4 till $0,98 \mathrm{mg} / \mathrm{kg} /$ dose. The concentration of PY ranged from 0,076 to $1,2 \mathrm{ug} /$ $\mathrm{ml}$. In 7 patients concentration value was below the therapeutic level (in 3 infants because of reduced dose of PY after neutropenia; in 3 the loading dose of PY was too low; in 1 technical error was made). In 3 cases concentration value was above therapeutic limits, despite recommended dose of the drug $(0,63 ; 0,55 ; 0,85 \mathrm{ug} / \mathrm{ml})$. Neutropenia was stated in 12 patients. In 10 patients the dose of PY was modified: because of neutropenia and/or hypertransaminasemia in 8 , and elevated level of $P Y$ in 2.

\section{Conclusions:}

1. Controlling of plasma concentration of pyrimethamine improve safety and effectiveness of therapy.

2. The measurement of pyrimethamine concentration in infants indicates the need of individualization of the drug dosage.

3. Neutropenia was the most common adverse effect during therapy. 